\title{
Switchbacks: statistical properties and deviations from Alfvénicity
}

\author{
A. Larosa ${ }^{1}$, V. Krasnoselskikh ${ }^{1,2}$, T. Dudok de Wit ${ }^{1}$, O. Agapitov $^{2}$, C. Froment ${ }^{1}$, V. K. Jagarlamudi ${ }^{1}{ }^{11}$, M. Velli ${ }^{13}$, \\ S. D. Bale ${ }^{2,4,5,6}$, A. W. Case ${ }^{7}$, K. Goetz ${ }^{8}$, P. Harvey ${ }^{2}$, J. C. Kasper ${ }^{12,7,9}$, K. E. Korreck ${ }^{7}$, D. E. Larson ${ }^{2}$, \\ R. J. MacDowall ${ }^{10}$, D. Malaspina ${ }^{3,14}$, M. Pulupa ${ }^{2}$, C. Revillet ${ }^{1}$, and M. L. Stevens ${ }^{7}$ \\ ${ }^{1}$ LPC2E, CNRS/University of Orléans/CNES, 3A avenue de la Recherche Scientifique, Orléans, France \\ e-mail: andrea.larosa@cnrs-orleans. fr \\ ${ }^{2}$ Space Sciences Laboratory, University of California, Berkeley, CA 94720-7450, USA \\ ${ }^{3}$ Laboratory for Atmospheric and Space Physics, University of Colorado, Boulder, CO 80303, USA \\ ${ }^{4}$ Physics Department, University of California, Berkeley, CA 94720-7300, USA \\ 5 The Blackett Laboratory, Imperial College London, London, SW7 2AZ, UK \\ ${ }^{6}$ School of Physics and Astronomy, Queen Mary University of London, London E1 4NS, UK \\ 7 Smithsonian Astrophysical Observatory, Cambridge, MA 02138, USA \\ ${ }^{8}$ School of Physics and Astronomy, University of Minnesota, Minneapolis, MN 55455, USA \\ ${ }_{9}$ Climate and Space Sciences and Engineering, University of Michigan, Ann Arbor, MI 48109, USA \\ 10 Solar System Exploration Division, NASA/Goddard Space Flight Center, Greenbelt, MD 20771, USA \\ ${ }^{11}$ National Institute for Astrophysics-Institute for Space Astrophysics and Planetology, Via del Fosso del Cavaliere 100, 00133 Roma, \\ Italy \\ ${ }^{12}$ BWX Technologies, Inc., Washington, DC 20002, USA \\ ${ }_{13}$ Department of Earth, Planetary, and Space Sciences, UCLA, Los Angeles, CA 90095, USA \\ 14 Astrophysical and Planetary Sciences Department, University of Colorado, Boulder, CO 80303, USA
}

Received 15 September 2020 / Accepted 15 January 2021

\begin{abstract}
Context. Parker Solar Probe's first solar encounter has revealed the presence of sudden magnetic field deflections in the slow Alfvénic solar wind. These structures, which are often called switchbacks, are associated with proton velocity enhancements.

Aims. We study their statistical properties with a special focus on their boundaries.

Methods. Using data from SWEAP and FIELDS, we investigate particle and wavefield properties. The magnetic boundaries are analyzed with the minimum variance technique.

Results. Switchbacks are found to be Alfvénic in $73 \%$ of cases and compressible in $27 \%$. The correlations between magnetic field magnitude and density fluctuations reveal the existence of both positive and negative correlations, and the absence of perturbations in the magnetic field magnitude. Switchbacks do not lead to a magnetic shear in the ambient field. Their boundaries can be interpreted in terms of rotational or tangential discontinuities. The former are more frequent.

Conclusions. Our findings provide constraints on the possible generation mechanisms of switchbacks, which have to be able to also account for structures that are not purely Alfvénic. One of the possible candidates, among others, manifesting the described characteristics is the firehose instability.
\end{abstract}

Key words. solar wind - magnetic fields - waves - magnetohydrodynamics (MHD)

\section{Introduction}

The Parker Solar Probe (PSP) mission (Fox et al. 2016), which was launched in 2018, offers a unique possibility to shed light on the main questions of solar physics: the heating of the solar corona and the acceleration of particles in the solar wind, by making in situ measurements in the extended solar corona. One of the most striking results of PSP is the omnipresence of sudden magnetic deflections that have been called jets, switchbacks, or velocity spikes (Bale et al. 2019; Kasper et al. 2019; Dudok de Wit et al. 2020; Horbury et al. 2020). Switchbacks had been observed before in fast streams of the polar solar wind by the Ulysses spacecraft (Balogh et al. 1999). They were recognized as folds in the field, which were distinguished from other in situ structures (Yamauchi et al. 2004b). Switchbacks were also observed in fast wind streams by Helios (Horbury et al. 2018) and were associated with one-sided radial jets (Gosling et al. 2009). An empiric model to explain their occurrence is proposed in Matteini et al. (2014). The first solar encounters of PSP show that they are ubiquitous features of the young slow solar wind. Therefore, their investigation should help us shed light on the dynamics and evolution of the young solar wind.

In switchbacks, the deflection of the magnetic field occurs simultaneously with that of the proton bulk velocity, which is also enhanced within the structures. These structures are considered to be highly Alfvénic in the sense that the magnitude of their magnetic field hardly varies in time. In addition, the magnetic 
field and proton velocity are highly correlated (Kasper et al. 2019). Several studies suggest that they are outward propagating Alfvénic structures (Bale et al. 2019; Kasper et al. 2019; Horbury et al. 2020), while others present evidence for long-range correlations and indicate that they are rooted deep down in the corona (Dudok de Wit et al. 2020). The distribution of the electron pitch angle indicates (Whittlesey et al. 2020) that switchbacks are not polarity changes, but they correspond to reversals of the same magnetic field line.

The radial Poynting flux associated with these structures represents approximately $10 \%$ of the kinetic energy flux (Bale et al. 2019). However, closer to the Sun, they might carry a more important fraction of energy flux because the ratio of Alfvénic flux to proton kinetic flux is inversely proportional to the Alfvénic Mach number, and so it increases close to the Sun, along with the magnetic field. The proton radial temperature is higher inside switchbacks (Kasper et al. 2019; Mozer et al. 2020; Krasnoselskikh et al. 2020). This correlation seems to be a detailed version of the more general strong correlation found between proton temperature and solar wind speed on larger scales (Grappin et al. 1990, 1991), except for structures with 180 degrees rotation (Woolley et al. 2020). Woodham et al. (2021) reveal that the parallel temperature is enhanced inside patches of switchbacks, while the perpendicular one is mostly constant, even though it is higher than the parallel one.

Switchback boundaries generally appear as strong discontinuities. The garnering interest in discontinuities in the solar wind goes back to the beginning of the space age (Burlaga 1968; Burlaga \& Ness 1969). Such discontinuities are accountable for a significant fraction of the wave power spectral density (Borovsky 2010). Their type determines the particle and energy exchange between plasma populations inside and outside localized regions such as magnetic field deflections. Phan et al. (2020) show the absence of reconnection at their boundaries and that the switchback boundaries of their data set rather behave as rotational discontinuities. On the contrary, Farrell (2020) has revealed the presence of magnetic dips and Froment et al. (2021) found some cases between 45 to 48 solar radii undergoing reconnection right at the boundaries. This latter result is particularly important because reconnection can quickly disintegrate the structures, thereby heating the background solar wind. Krasnoselskikh et al. (2020), in case studies, point out the presence of strong currents at these boundaries and an enhanced radial Poynting flux. This suggests that some boundary regions may be unstable with respect to the Kelvin-Helmholtz instability (Kasper et al. 2019; Mozer et al. 2020). Finally, these boundaries show enhanced wave activity ranging from Magnetohydrodynamics (MHD) scales (as we are going to show) to the whistler frequency range $(50-150 \mathrm{~Hz}$ ) (Agapitov et al. 2020; Jagarlamudi et al. 2021) and beyond. There are still many open questions regarding the nature and origin of switchbacks, with a special interest in their role in the solar wind energy balance and plasma heating. Here we address the following questions:

- What is the proportion of Alfvénic versus compressional structures?

- What are the sources of the different types of structures and what instabilities could be responsible for their generation?

- What are the characteristics of their boundaries and is there any connection between them and the discontinuities that are observed farther away from the Sun (by other missions)?

To answer these questions, we hereafter carry out a statistical study of the characteristics of these structures by making use of a manually selected set. This paper is organized as follows: in Sect. 2 we present the data and the methods; following that, we present a statistical analysis of the main properties of switchbacks in Sect. 3, the statistical characteristics of boundaries are presented in Sect. 4, and they are summarised in Sect. 5. We conclude in Sect. 6.

\section{Data and methods}

We used the DC magnetic field from the fluxgate magnetometer (MAG) and the AC magnetic field from the search coil magnetometer (SCM); both instruments are part of the FIELDS suite (Bale et al. 2016). The proton density, velocity, and temperature were derived from the ion distribution function, which was measured by the Faraday cup which is part of the SWEAP suite (Kasper et al. 2016). The electron density was estimated from the quasi thermal noise technique (Moncuquet et al. 2020). We used the RTN coordinate system throughout the paper: the $R$ component is directed anti-sunward along the Sun-spacecraft direction; the tangential $T$ component is the cross product of the solar rotation vector with $R$; and the normal $N$ component completes the right-handed set and points in the same direction as the solar rotation vector.

We identified 70 switchbacks with sharp boundaries between 3 November 2018 and 8 November 2018. During this period, the cadence of the Faraday cup was of $0.87 \mathrm{~s}$, while MAG and SCM were sampled either at 146 or at $293 \mathrm{~Hz}$. The identification of the switchbacks was made based on the four basic characteristics that are common to all of them: a deflection from the anti-sunward direction of the magnetic field, coming back to the initial conditions, an increase in the magnetic fluctuation amplitude registered by SCM and MAG, and an increase in the proton bulk velocity and radial temperature (this is the only thermal characteristic that is routinely provided by the SWEAP suite Kasper et al. 2016 at the moment).

Figure 1 shows a typical switchback signature in the magnetic field from MAG. For each structure, we recorded the median magnetic field from MAG and the magnitude of the magnetic field fluctuations from SCM; the velocity, the density, and the temperature in the regions indicated in the figure as "Before", "Inside", and "After" to compare the plasma properties of the structure with respect to the surrounding plasma.

We study the boundary type, geometry, thickness, and current density by applying the minimum variance analysis technique (MVA) (Sonnerup \& Scheible 1998) to MAG data. MVA has been extensively used to analyze solar wind discontinuities and current sheet crossings and its limits have been evaluated and validated by comparison with a triangulation estimation of the same quantities (Horbury et al. 2001; Knetter 2004). In spite of its limitations, MVA is a valuable tool for investigating the geometry and the nature of the discontinuities dealing with single point measurements. Here, for each structure that matches the four abovementioned conditions, we applied the technique to its leading and trailing edges.

The application of the MVA is sensitive to the chosen interval around the transition and can be affected by the presence of waves or spike-like structures that are ubiquitous at switchback boundaries. For that reason, it is difficult to perform an automated analysis and (subjective) user intervention is often needed. To avoid complex transitions, we only consider cases for which the ratio between intermediate and minimum eigenvalues exceeds two (Lepping \& Behannon 1980), although a ratio higher than ten has been recommended (Knetter 2004) to avoid errors due to anisotropic 3D wave activity or surface waves. To further attenuate the impact of wave activity and avoid measuring the wavenumber vector of waves rather than the normal to 


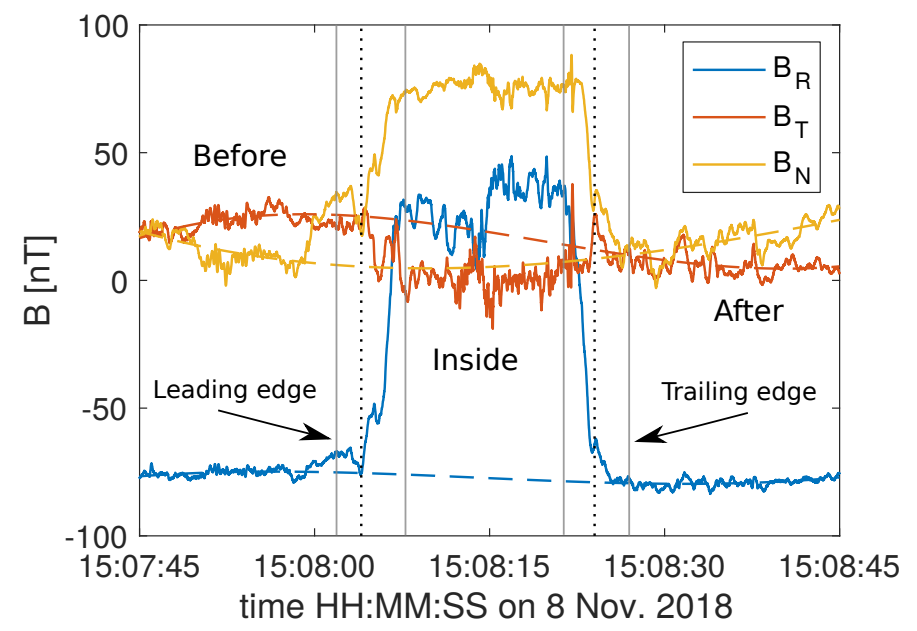

Fig. 1. Typical switchback with a strong deflection and well defined edges. The leading and trailing edges are found between the gray vertical lines. Dashed lines represent the slow evolution of the magnetic field as if no switchbacks were occurring; they are also meant to reveal a potential departure of the magnetic field from that trend when approaching the switchback. These trends were obtained from a third order polynomial approximation that was adjusted to the magnetic field up to $5 \mathrm{~s}$ before the onset of the leading edge of the switchback and starting again $5 \mathrm{~s}$ after the end of the trailing edge. These transitions are indicated by vertical dotted lines.

the boundary (see for example Hudson 1970), we low-pass filtered the magnetic field data, removing frequencies above a few Hertz when needed. Another issue is the presence of transient spikes at the edges, as has already been shown by Kasper et al. (2019). When present, they can mislead the MVA. We removed them with a median filter that was tuned on a case by case basis. Figure 2 illustrates one example in which the duration of the spike is approximately $2 \mathrm{~s}$, while the duration of the leading edge is more than one minute. Here, although the spike is a shortlived transient, it may nevertheless alter the result of the MVA analysis. In situations such as this, we applied the median filter, whose result is shown in the lower panel of Fig. 2. In the interpretation of the switchbacks as magnetic flux tube crossings (Krasnoselskikh et al. 2020), such transients are precisely the signature of wave activity occurring at the surface of the tubes. Here, however, we discarded them because our objective is to estimate the normal to the boundary.

\section{Main properties of switchbacks}

Typical signatures of a switchback are shown in Fig. 3. This particular structure has an almost constant magnetic field magnitude, lower proton density inside, increased velocity in both radial and normal components, enhanced wave activity, and radial temperature inside. The duration is $130 \mathrm{~s}$, while the leading edge lasts $14 \mathrm{~s}$ and the trailing edge lasts $20 \mathrm{~s}$.

In Fig. 4 we summarize the main statistical properties of the switchbacks with respect to the surrounding plasma. The first plot represents the distribution of average magnetic field magnitude inside relative to the average magnetic field magnitude outside. We note that the large fraction of Alfvénic structures, corresponding to the peak at a ratio of one, is surrounded by a population of compressible structures. The presence of both types of structures had already been suggested by Bale et al. (2019) and Krasnoselskikh et al. (2020). If we consider structures that are located between 0.95 and 1.05 to be Alfvénic, then

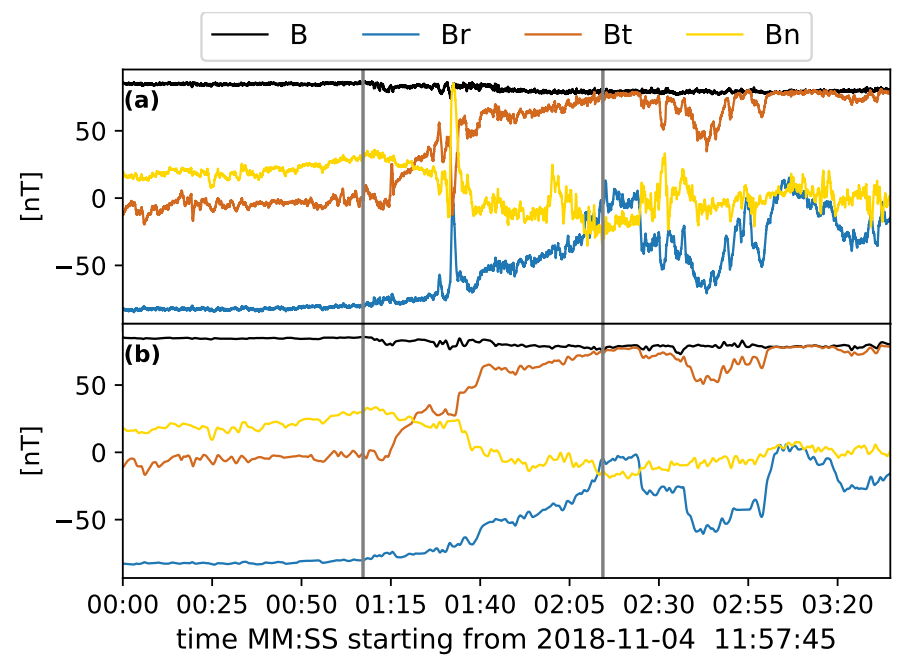

Fig. 2. Example of a spike at the leading edge of a switchback. Upper panel: three components and the amplitude of the original MAG data. Lower panel: same data after running a median filter with a 10 -s window (five times the duration of the spike) and low-pass filtering. The leading edge of the switchback is located between the gray vertical lines.

$73 \%$ are Alfvénic and $27 \%$ are compressible. The second plot illustrates the same ratio of velocities inside/outside and reveals a net increase that is on the order of $100 \mathrm{~km} \mathrm{~s}^{-1}$, which is comparable to the Alfvén velocity. The proton density (third plot) and the electron density (not shown) do not show any preferential variation. The magnitude of broadband magnetic fluctuations registered by the SCM (fourth plot) is generally higher inside, except in four cases, with only two cases for which the ratio is below 0.98 . The largest relative increases in wave activity are observed for those few isolated switchbacks that are embedded in a quiet interval with an almost radial field. The plasma radial $\beta$ (i.e., $\beta$ computed using the radial temperature) is systematically higher inside than outside, as shown in the fifth plot. When plotted as a function of the deflection angle of the magnetic field across the boundaries, this ratio peaks at around 90 degrees. This dependence suggests that higher values of $\beta$ may be caused by a temperature anisotropy rather than by higher temperatures inside the switchbacks. On the contrary, if we compute the same $\beta$ ratio by artificially keeping a constant temperature across the structures, we still find an enhancement of $\beta$ at the interior of the structures. In this case, the median value of the ratio decreases from 1.45 to 1.26. In Fig. 5, we plotted the magnetic field ratio against the density ratio. Non-Alfvénic structures lie outside the dashed lines. For ten structures the density and the magnetic field are anticorrelated and for nine they are correlated. This correlation between density and magnetic field variations can be used for wave mode identification. Indeed, in isotropic and homogeneous plasma, such correlations are known to correspond to fast magnetosonic waves when positive and to slow mode when negative (Stix 1992). This important property was used by Chaston et al. (2020) in their study of wave decomposition into eigenmodes obtained in the MHD approximation; their study is based on observations made by PSP during its first encounter for frequencies ranging from 0.0002 to $0.2 \mathrm{~Hz}$. The authors find the major wave mode to belong to shear Alfvén waves, with approximately $20-30 \%$ of the energy carried by slow mode waves. It is worth noting that they excluded switchback boundaries form their analysis and thus did not identify which wave mode should be attributed to the switchbacks themselves. 


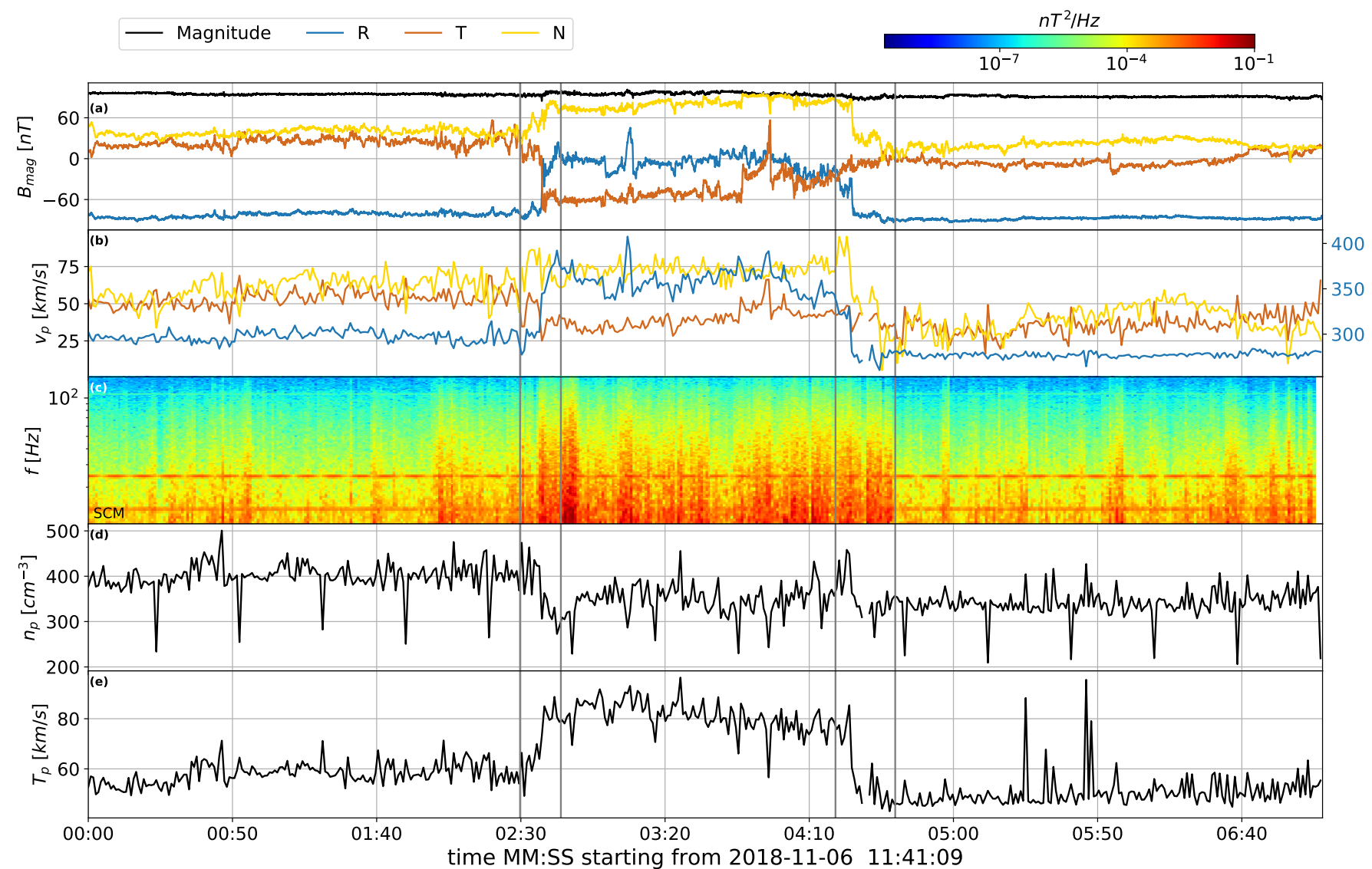

Fig. 3. Switchback example. Panel a: MAG magnetic field data; $(b)$ : proton velocity; $(c)$ : trace spectrogram from the SCM waveform; $(d)$ : proton density; and (e): proton temperature. Leading and trailing edges are located between the first and second pairs of vertical lines, respectively.

The correlation between density and magnetic variations does not show any dependence on the deflection angle of the switchback. We note that the less structured and shorter-lived switchbacks are mostly Alfvénic.

Another question of interest is whether switchbacks modify the magnetic field in their vicinity, for example, because of currents flowing in or along it. Quantifying such a distortion is challenging because it requires switchbacks with sharp edges that are surrounded by a quiet magnetic field without trends or evidence of other or embedded switchbacks. We found only a few tens of such cases during the first encounter of PSP. Figure 1 illustrates one of them, which in addition has a strong deflection of more than $90^{\circ}$. To determine a possible distortion, we extrapolated the magnetic field during the switchback by considering its value up to $5 \mathrm{~s}$ before the onset of the leading edge and again $5 \mathrm{~s}$ after the end of the trailing edge. The figure shows scarce evidence for a distortion in the magnetic field near the edges. We performed the same analysis on 16 switchbacks with sharp edges and a reversal of more than 90 degrees and only two showed weak evidence for a distortion. We conclude that the current flowing at the boundary of switchbacks does not affect the surrounding magnetic field in a significant way.

The transverse size of switchbacks can be estimated from the product of the normal velocity (the projection of the proton velocity along the normal direction $\hat{n}$ ) multiplied by the crossing time. Since there are two different normal velocities at leading and trailing edges, we first computed the size of both edges. We evaluated the size of the whole structure as the sum of the two edges plus the size of the internal region. To estimate the latter, we used the average of the velocities at the leading and trailing edges multiplied by the crossing time. The results are shown in Fig. 6. The size of the largest structure in our data set is approximately $0.65 R_{\text {sun }}$. The sizes of the boundaries exceed the ion inertial length between one and three orders of magnitude and the structures may therefore be interpreted in terms of MHD discontinuities.

In making use of the technique presented by Krasnoselskikh et al. (2020), we estimated the currents from the rotation of the magnetic field between both sides of each boundary. At MHD scales, we have $\boldsymbol{J}=\boldsymbol{\nabla} \times \boldsymbol{B} / \mu_{0}$. Moving across a switchback boundary, the spatial variation happens along the minimum variance direction, while the component of the field that has a considerable gradient is the maximum variance component. Therefore, the cross product of the two that is along the intermediate variance direction should give an estimate of the current. A similar approach was also used in Artemyev et al. (2018). The result of this procedure is shown in Fig. 7. The currents we find are between one and two orders of magnitude larger than those observed in solar wind discontinuities at $1 \mathrm{AU}$ (Artemyev et al. 2018), even though the sizes of the discontinuities are comparable. The main difference between the two resides in the stronger magnetic field at the location of PSP.

\section{Boundaries}

The results of our MVA analysis of switchback boundaries are summarized in Fig. 8. Here we use the same classification scheme as Neugebauer et al. (1984) and Horbury et al. (2001) for both leading and trailing edges combined together in order to have a better statistics. 

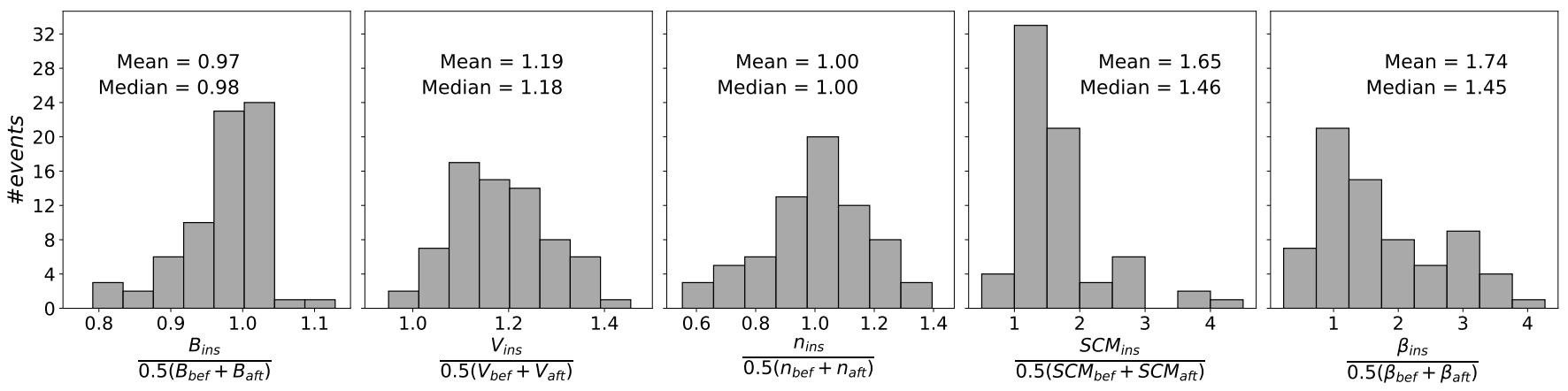

Fig. 4. Main statistical properties of switchbacks, showing their average value inside of the switchback relative to that observed outside for different quantities, see Fig. 1. From left to right: magnetic field magnitude, proton velocity, proton density, amplitude of the magnetic field fluctuations as measured from SCM, and radial plasma $\beta$.

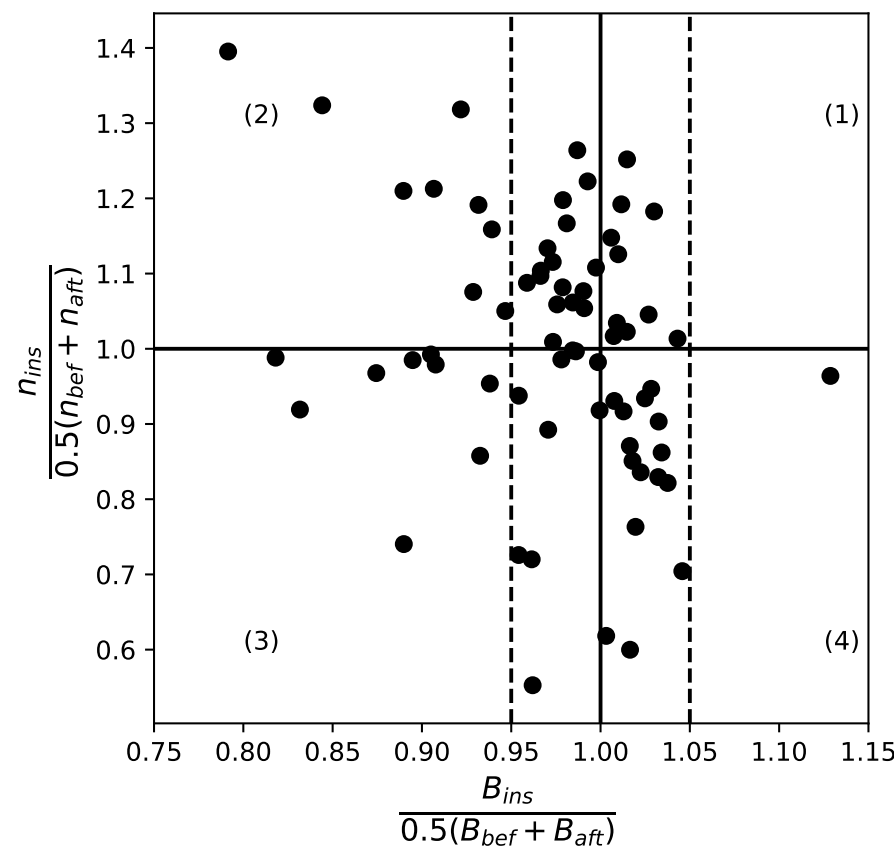

Fig. 5. Comparison of magnetic field and density with ratios of values inside/outside switchbacks. The black vertical and horizontal lines split the values into four quadrants. Quadrants (1) and (3) correspond to structures in which fluctuations are positively correlated, and quadrants (2) and (4) correspond to anticorrelated fluctuations. We consider the structures that are located between the two vertical dashed lines to be Alfvénic.

Discontinuities with $\boldsymbol{B} \cdot \hat{n} /|B|>0.4$ and $\delta|B| /|B|<0.2$ are supposedly rotational discontinuities (RDs) because they have a significant field component normal to the surface (considered as a plane), while the magnitude of the variation is still consistent with Alfvénic fluctuations. Discontinuities with $\boldsymbol{B} \cdot \hat{n} /|B|<0.4$ and $\delta|B| /|B|>0.2$ are classified as tangential discontinuities (TDs) because they present a major change in magnetic field magnitude while the field threading does not represent a large fraction of the total field. The area denoted by "Either" (ED) corresponds to values of parameters for which it is difficult to unambiguously distinguish between rotational and tangential discontinuities; the area marked as "Neither" (ND) is inconsistent with characteristics of the MHD discontinuities and indeed is mostly empty.

We found that $32 \%$ of the switchback boundaries are RDs, $17 \%$ are TDs, $42 \%$ are in the "Either" area, and $9 \%$ are in the
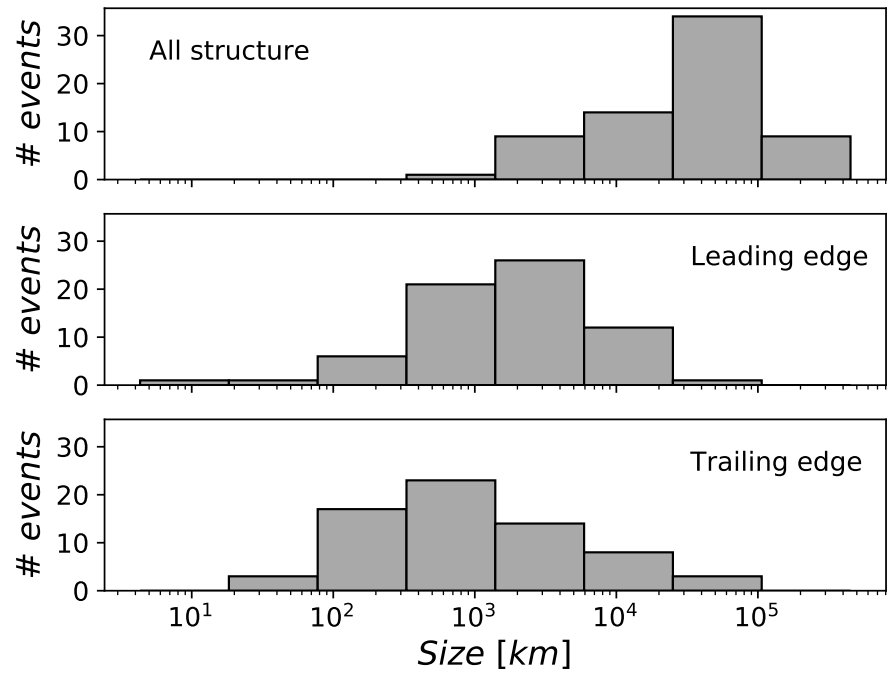

Fig. 6. Distribution of the sizes of the switchback edges and full structure.
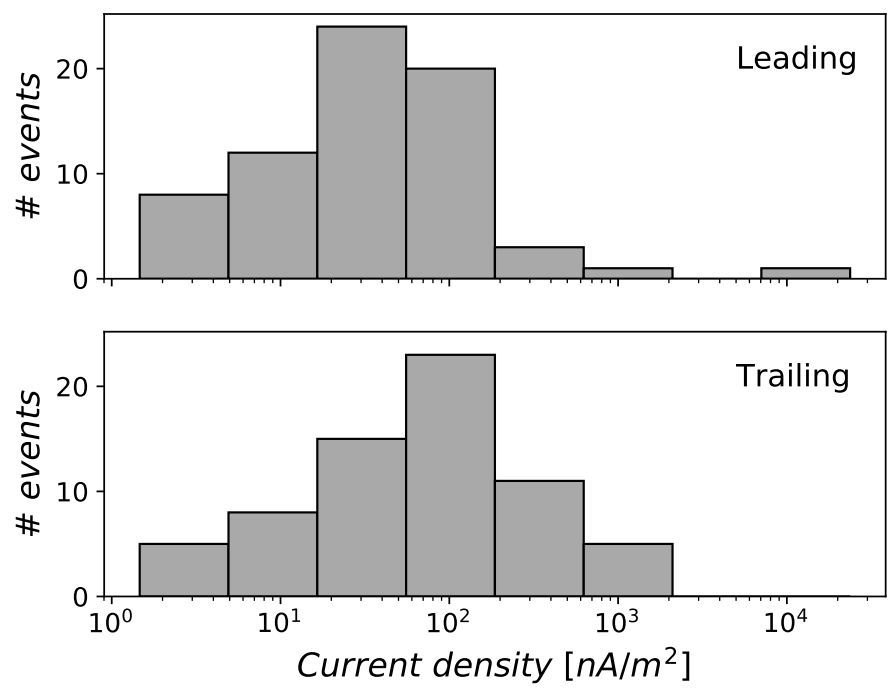

Fig. 7. Distribution of the magnitude of the current density at switchback boundaries.

"Neither" area. These results are consistent with similar studies performed at 1 AU (see Table 2 of Neugebauer 2006). The higher occurrence of RDs with respect to TDs is expected for Alfvénic winds emerging from coronal holes (Söding et al. 2001). 


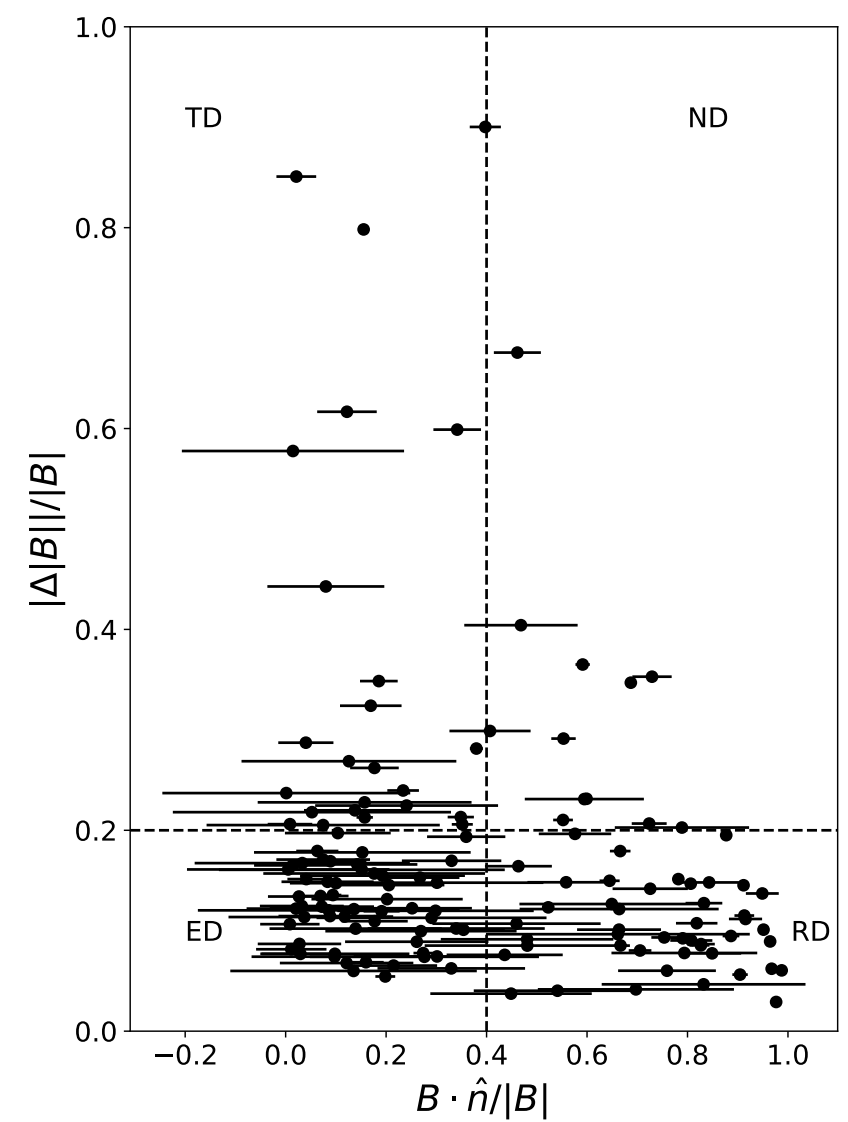

Fig. 8. Boundary classification comparing the field threading through the discontinuity surface normalized to the field magnitude ( $x$ axis) and the highest median magnetic field magnitude between the two sides of the discontinuity ( $y$ axis). The dotted lines separate different types of discontinuities. The $\pm 1 \sigma$ error bars in the $x$ direction were estimated by block bootstrapping.

We note that periods when $\delta|B| /|B|$ is above $40 \%$ are typically due to sharp dips in the magnetic field that happen right at the switchback boundaries. The field quickly recovers its magnitude once the boundary is crossed. This is the reason why such sharp changes are not observed in the first distribution of Fig. 4. The structures for which $\boldsymbol{B} \cdot \hat{n} /|B|$ is close to one are switchbacks in which the MVA direction is close to the radial direction. For that reason, they have a large normal component. This happens for Alfvénic fluctuations that propagate nearly parallel to the ambient magnetic field (Söding et al. 2001). TDs can be generated either at the stream interface by the mirror instability (Söding et al. 2001) or by the diamagnetic component of boundaries current (Krasnoselskikh et al. 2020; Farrell 2020).

This gives two scenarios for switchbacks that are embedded between two TDs. In the first one, since no significant velocity shear is observed across the boundaries, the switchback has to be generated deeper down in the corona where supposedly the shear occurred. In the second case, the TDs would be just the result of the mirror instability at switchback boundaries or the effect of the diamagnetic currents.

RDs could be the steepened edge of a large amplitude Alfvén waves (Tsurutani et al. 1994), a picture that is consistent with the constant magnetic field magnitude switchbacks with rotational discontinuities as boundaries. However, in most cases, we find the type of the discontinuity to be different at the leading and trailing edges. Most switchbacks have a TD at their leading edge and $\mathrm{RD}$ at their trailing edge, or vice versa.

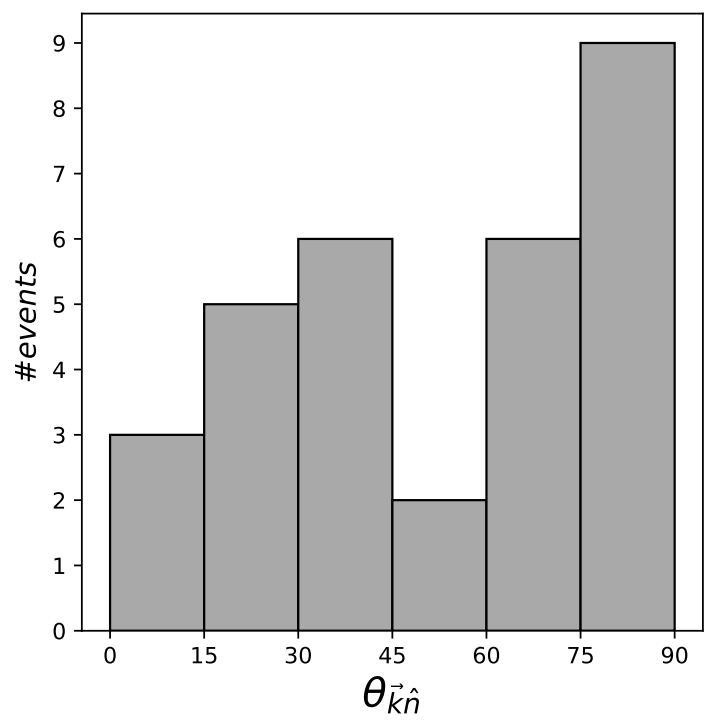

Fig. 9. Distribution of the angles between the normal to the boundary and the $k$ vector of the wave located at that boundary.

The MAG and SCM magnetometers often reveal enhanced levels of magnetic wave activity at switchback boundaries. Most waves are broadband with frequencies typically ranging between 0.1 and $30 \mathrm{~Hz}$. To analyze these waves, we bandpass filtered them and performed a polarization analysis (Santolík et al. 2003) in the frequency band that has the largest power. This analysis was complemented by a visual inspection of the hodograms. In the following, we only consider those waves whose polarity exceeds 0.7. Of the 140 switchback boundaries, we analyzed only 32 which present polarized waves matching our criteria. Most of these waves have frequencies around $3 \mathrm{~Hz}$. The angle between the normal to the boundary and their wavenumber vector (see Fig. 9) is restricted between 0 and 90 degrees due to the intrinsic ambiguity of the polarization analysis method. With this, we find the median value to be approximately 60 degrees; this large angle is consistent with the presence of surface waves as suggested by Krasnoselskikh et al. (2020). Waves propagating more parallel to the boundary normal are probably gradient drift waves that are generated by the gradients of the plasma parameters across switchback boundaries.

\section{Summary of observations}

We have presented a statistical study of the characteristics of the switchbacks and their boundaries. Their characteristic transverse sizes vary from several thousands of kilometers up to the solar radius. Some of them, mainly small-scale ones, are quite uniform, while others have well pronounced internal substructures. We have shown the presence of two different types of switchbacks, Alfvénic and compressible. The former are more frequent than the latter. Our study also reveals that the majority of switchbacks present important plasma density variations from outside to inside. The density can either increase or decrease, but typically it does not remain the same as outside. We found that the density and magnetic field jumps are positively correlated for some switchbacks and anticorrelated for others, but most of them present density jumps even when the magnetic field strength remains practically constant. These density jumps were observed with both SPAN-i and SPC. The wave activity is enhanced for the majority of switchbacks at their interior and for a few of them at their boundaries, where surface waves are probably present. 
The radial $\beta$ is enhanced inside the structures. The investigation of switchback boundaries led us to their interpretation in terms of MHD discontinuities since their size is orders of magnitude larger than the ion inertial length and ion Larmor radius. The classification of the boundaries shows the presence of both rotational and tangential discontinuities, the former being twice more frequent. Strong currents occur at the boundaries of switchbacks; this corroborates the idea proposed by Krasnoselskikh et al. (2020) that these structures are localized kinked magnetic tubes separated from surrounding plasma by surface currents.

\section{Discussion and conclusions}

Our statistical study aims to provide possible constraints imposed by the characteristics of switchbacks, which may in turn help determine the possible generation mechanisms of these structures and their possible role in heating and accelerating the solar wind. The large variety of scales and characteristics suggests that several mechanisms may generate switchbacks. To the best of our knowledge, four mechanisms have been proposed so far.

The first mechanism was originally proposed to explain observations made by Ulysses of so-called interchange reconnection between closed and open magnetic field lines (Yamauchi et al. 2004a). More recently, this explanation has been invoked by Fisk \& Kasper (2020) to explain the generation of switchbacks at the boundary of the small coronal holes due to reconnection between open and closed field lines. This transient reconnection supposedly occurs in low- $\beta$ plasmas. Following this, Alfvénic and slow-mode-type perturbations propagate along open field lines, generating a helicity that is eventually evacuated by means of convection to interplanetary space in the form of Alfvénictype perturbations (see Edmondson 2012). Such processes may naturally occur inside and at the boundary of coronal holes (Yamauchi et al. 2004a; Fisk 2003). Numerous switchbacks were indeed observed by PSP during its first solar encounters whenever the spacecraft was connected to small coronal holes (Badman et al. 2020; Panasenco et al. 2020). In contrast, very few switchbacks occurred during the second encounter, when there was no such connection. The characteristics of the structures we identified are consistent with such a mechanism. In particular, interchange reconnection may explain the Alfvénic and compressible structures that correspond to the anticorrelation of density and magnetic field variations. Fast-mode-like structures are unlikely to arise with interchange reconnection (Kigure et al. 2010). A second plausible mechanism arises from the evolution of Alfvén waves propagating in the expanding solar wind (Hollweg 1974; Velli 1993; Landi et al. 2006). In the presence of radial gradients, the amplitude of fluctuations grows relative to the mean field, so that spherically polarized Alfvén waves with a small radial component may grow until a switchback is formed. This mechanism was demonstrated by means of computer simulations based on the expanding box model (Velli et al. 1992; Grappin \& Velli 1996; Squire et al. 2020). It gives rise to a predominant population of Alfvénic modes, with small slow or fast contributions depending on the plasma $\beta$. However, we are lacking information about the range of sizes of the structures that may be generated this way.

A third possibility is the interaction of shear flows, which are naturally present in the young solar wind, with the omnipresent fluctuations that propagate away from the Sun. This mechanism was first proposed by Landi et al. $(2005,2006)$. A slightly different version, involving the nonlinear instability of such sheared flows, has been proposed by Ruffolo et al. (2020). This mechanism mainly leads to a slow-mode (pressure-balanced) contribution to the switchback. Such structures exhibit an anticorrelation between the density and magnetic field variations, which is in agreement with our observations. However, they are expected to be less frequent than Alfvénic structures.

Finally, a fourth mechanism is the generation of perpendicular magnetic perturbations due to microscopic plasma instabilities. Indeed, the excess of parallel streaming due to either pressure anisotropy or to the presence of a secondary beam can lead to structures that are similar to switchbacks. Tenerani \& Velli (2018), using simulations, show how magnetic field reversals bounded by rotational discontinuities can be a natural consequence of the nonlinear evolution of right-handed circularly polarized Alfvén waves in high- $\beta$ plasma regimes that are unstable with respect to the firehose instability. The proton temperature anisotropy measured by PSP is not favorable to trigger such an instability as proton beams are mostly observed in quiet radial field regions (Verniero et al. 2020). Nevertheless, remote sensing observations show the presence of numerous jets in the lower corona (Sterling \& Moore 2020). The velocity of these jets may exceed the Alfvén velocity, thus offering favorable conditions for the firehose instability.

Another possible source of instability of right-handed polarized Alfvén waves may be related to the presence of alpha particles. These particles show a tendency to have $T_{\|}>T_{\perp}$ moving towards the Sun (Stansby et al. 2019) and numerical simulations have shown that they can trigger the firehose instability (Matteini 2015). We note that a firehose-like mode could also grow by the cyclotron resonant interaction of a parallel propagating wave of the fast magnetosonic-whistler branch with alpha particles in the presence of an alpha-proton drift equal to or greater than 1.7 Alfvén speeds (Verscharen \& Chandran 2013). The oblique firehose instability initially gives rise to non-propagating modes with anticorrelated magnetic and density fluctuations; these modes eventually turn into elliptically polarized Alfvén waves (Hellinger \& Matsumoto 2000). This mechanism could produce the slow mode-like anticorrelation observed for some of the switchbacks. It is worth noting that the microscopic plasma instabilities may account for small- and moderate-scale perturbations, though it is difficult to determine their limiting scale because of the effect of the solar wind expansion.

The presence of Alfvénic, fast, and slow mode signatures in switchbacks is important to determine the most plausible generation mechanisms. There is a caveat, however. Our analysis does not unambiguously attribute the structures to a certain type of wave mode, since it is only based on the correlation and anticorrelation of the density perturbations and magnetic field magnitude. As shown by Hau \& Sonnerup (1993), in anisotropic plasmas, fast modes always show a positive correlation, whereas slow modes can lead to both positive and negative correlations depending on parameters, such as the angle between the wavenumber vector and the magnetic field, the level of anisotropy, and the chosen state equation for moments closure. Of particular interest is their result, showing that under anisotropic conditions, favorable to the firehose instability, slow modes exhibit a positive correlation for a wider range of angles between the magnetic field and wavenumber. From this, we conclude that the only two mechanisms that are able to explain all three types of observed modes are the evolution of Alfvén waves in the expanding solar wind and firehose-like instabilities.

Acknowledgements. The FIELDS experiment was developed and is operated under NASA contract NNN06AA01C. A.L., V.K., T.D., C.F., V.K.J. 
and C.R. acknowledge financial support of CNES in the frame of Parker Solar Probe grant. S.D.B. acknowledges the support of the Leverhulme Trust Visiting Professorship programme. O.A. and V.K. were supported by NASA grant 80 NSSC20K0697. O.A. was partially supported by NASA grants 80NNSC19K0848, 80NSSC20K0218, and NSF grant NSF 1914670. Parker Solar Probe was designed, built, and is now operated by the Johns Hopkins Applied Physics Laboratory as part of NASA's Living with a Star (LWS) program (contract NNN06AA01C). Support from the LWS management and technical team has played a critical role in the success of the Parker Solar Probe mission. The data used in this study are available at the NASA Space Physics Data Facility (SPDF), https://spdf.gsfc.nasa.gov.

\section{References}

Agapitov, O. V., Dudok de Wit, T., Mozer, F. S., et al. 2020, ApJ, accepted Artemyev, A. V., Angelopoulos, V., Halekas, J. S., et al. 2018, ApJ, 859, 95 Badman, S. T., Bale, S. D., Martínez Oliveros, J. C., et al. 2020, ApJS, 246, 23

Bale, S. D., Goetz, K., Harvey, P. R., et al. 2016, Space Sci. Rev., 204, 49

Bale, S. D., Badman, S. T., Bonnell, J. W., et al. 2019, Nature, 576, 237

Balogh, A., Forsyth, R. J., Lucek, E. A., Horbury, T. S., \& Smith, E. J. 1999, Geophys. Res. Lett., 26, 631

Borovsky, J. E. 2010, Phys. Rev. Lett., 105, 111102

Burlaga, L. F. 1968, Sol. Phys., 4, 67

Burlaga, L. F., \& Ness, N. F. 1969, Sol. Phys., 9, 467

Chaston, C. C., Bonnell, J. W., Bale, S. D., et al. 2020, ApJS, 246, 71

Dudok de Wit, T., Krasnoselskikh, V. V., Bale, S. D., et al. 2020, ApJS, 246, 39

Edmondson, J. K. 2012, Space Sci Rev., 172, 209

Farrell, W. M. 2020, ApJS, 10

Fisk, L. A. 2003, J. Geophys. Res., 108, 1157

Fisk, L. A., \& Kasper, J. C. 2020, ApJ, 894, L4

Fox, N. J., Velli, M. C., Bale, S. D., et al. 2016, Space Sci Rev., 204, 7

Froment, C., Krasnoselskikh, V., Dudok de Wit, T., et al. 2021, A\&A, 650, A5 (PSP SI)

Gosling, J. T., McComas, D. J., Roberts, D. A., \& Skoug, R. M. 2009, ApJ, 695, L213

Grappin, R., \& Velli, M. 1996, J. Geophys. Res. Space Phys., 101, 425

Grappin, R., Mangeney, A., \& Marsch, E. 1990, J. Geophys. Res., 95, 8197

Grappin, R., Velli, M., \& Mangeney, A. 1991, Ann. Geophys., 9, 416

Hau, L. N., \& Sonnerup, B. U. O. 1993, Geophys. Res. Lett., 20, 1763

Hellinger, P., \& Matsumoto, H. 2000, J. Geophys. Res. Space Phys., 105, 10519

Hollweg, J. V. 1974, J. Geophys. Res., 79, 1539

Horbury, T. S., Burgess, D., Fränz, M., \& Owen, C. J. 2001, Geophys. Res. Lett., 28,677

Horbury, T. S., Matteini, L., \& Stansby, D. 2018, MNRAS, 478, 1980

Horbury, T. S., Woolley, T., Laker, R., et al. 2020, ApJS, 246, 45
Hudson, P. 1970, Planet. Space Sci., 18, 1611

Jagarlamudi, V. K., Dudok de Wit, T., Froment, C., et al. 2021, A\&A, 650, A9 (PSP SI)

Kasper, J. C., Abiad, R., Austin, G., et al. 2016, Space Sci. Rev., 204, 131

Kasper, J. C., Bale, S. D., Belcher, J. W., et al. 2019, Nature, 576, 228

Kigure, H., Takahashi, K., Shibata, K., Yokoyama, T., \& Nozawa, S. 2010, PASJ, 62,993

Knetter, T. 2004, J. Geophys. Res., 109, A06102

Krasnoselskikh, V., Larosa, A., Agapitov, O., et al. 2020, ApJ, 893, 93

Landi, S., Hellinger, P., \& Velli, M. 2005, ESA SP, 592, 785

Landi, S., Hellinger, P., \& Velli, M. 2006, Geophys. Res. Lett., 33, L14101

Lepping, R. P., \& Behannon, K. W. 1980, J. Geophys. Res. Space Phys., 85, 4695

Matteini, L. 2015, ApJ, 802, 11

Matteini, L., Horbury, T. S., Neugebauer, M., \& Goldstein, B. E. 2014, Geophys. Res. Lett., 41, 259

Moncuquet, M., Meyer-Vernet, N., Issautier, K., et al. 2020, ApJS, 246, 44

Mozer, F. S., Agapitov, O. V., Bale, S. D., et al. 2020, ApJS, 246, 68

Neugebauer, M. 2006, J. Geophys. Res., 111, A04103

Neugebauer, M., Clay, D. R., Goldstein, B. E., Tsurutani, B. T., \& Zwickl, R. D. 1984, J. Geophys. Res., 89, 5395

Panasenco, O., Velli, M., D’Amicis, R., et al. 2020, ApJS, 246, 54

Phan, T. D., Bale, S. D., Eastwood, J. P., et al. 2020, ApJS, 246, 34

Ruffolo, D., Matthaeus, W. H., Chhiber, R., et al. 2020, ApJ, 902, 94

Santolík, O., Parrot, M., \& Lefeuvre, F. 2003, Radio Sci., 38

Söding, A., Neubauer, F. M., Tsurutani, B. T., Ness, N. F., \& Lepping, R. P. 2001, Ann. Geophys., 19, 667

Sonnerup, B. U. Ö., \& Scheible, M. 1998, ISSI Sci. Rep. Ser., 1, 185

Squire, J., Chandran, B. D. G., \& Meyrand, R. 2020, ApJ, 891, L2

Stansby, D., Perrone, D., Matteini, L., Horbury, T. S., \& Salem, C. S. 2019, A\&A, 623, L2

Sterling, A. C., \& Moore, R. L. 2020, ApJ, 896, L18

Stix, T. H. 1992, Waves in Plasmas (American Institute of Physics)

Tenerani, A., \& Velli, M. 2018, ApJ, 867, L26

Tsurutani, B. T., Ho, C. M., Smith, E. J., et al. 1994, Geophys. Res. Lett., 21, 2267

Velli, M. 1993, A\&A, 270, 304

Velli, M., Grappin, R., \& Mangeney, A. 1992, AIP Conf. Proc., 267, 154

Verniero, J. L., Larson, D. E., Livi, R., et al. 2020, ApJS, 248, 5

Verscharen, D., \& Chandran, B. D. G. 2013, ApJ, 764, 88

Whittlesey, P. L., Larson, D. E., Kasper, J. C., et al. 2020, ApJS, 246, 74

Woodham, L. D., Horbury, T. S., Matteini, L., et al. 2021, A\&A, 8

Woolley, T., Matteini, L., Horbury, T. S., et al. 2020, MNRAS, 498, 5524

Yamauchi, Y., Moore, R. L., Suess, S. T., Wang, H., \& Sakurai, T. 2004a, ApJ, 605, 511

Yamauchi, Y., Suess, S. T., Steinberg, J. T., \& Sakurai, T. 2004b, J. Geophys. Res. Space Phys., 109, A03104 